\title{
DETERMINANTES DOS MOVIMENTOS PENDULARES NO BRASIL: UMA ANÁLISE ESPACIAL $^{\circ}$
}

\author{
DETERMINANTS OF COMMUTING IN BRAZIL: \\ A SPATIAL ANALYSIS \\ Danyella Juliana Brito* \\ Marcus Vinícius Amaral e Silva** \\ enviado: 05 febrero 2020 - aceptado: 07 septiembre 2020
}

\begin{abstract}
Resumo
As decisões de onde viver e trabalhar envolvem dilemas entre oportunidades, salários, tempo de deslocamento e custo de vida. O objetivo do artigo é analisar quais os fatores municipais associados com as maiores taxas de deslocamento pendular, nos municípios brasileiros em 2010, compreendendo esses movimentos como possivelmente autocorrelacionados espacialmente. Para tanto, utilizaram-se técnicas de econometria espacial. Dentre os principais achados está a necessidade da ponderação da autocorrelação espacial na análise dos determinantes da mobilidade pendular. Ademais, os resultados indicam que variáveis de mercado de trabalho e de aglomeração urbana estão diretamente associadas aos maiores fluxos de entrada de pendulares. Por outro lado, as características de infraestrutura urbana não parecem explicar a pendularidade pelas mesmas vias que explica a atratividade migratória.

Palavras Chave: mercado de trabalho, deslocamento pendular, análise espacial Classificação JEL: J61, R10, C31.

Brito, D. J. M., \& Amaral e Silva, M. V. (2021). Determinantes dos movimentos pendulares no Brasil: uma análise espacial. Estudios económicos, 38(76), pp 95-122.

* Universidade Federal de Pernambuco, Brasil. E-mail: danyella.brito@ufpe.br. ORCID: https://orcid. org/0000-0002-9630-2577.

** Universidade Federal do Piauí, Brasil. E-mail: vinicius.amaral@ufpi.br. ORCID: https://orcid. org/0000-0002-9361-9448.
\end{abstract}




\begin{abstract}
Decisions about where to live and work involve dilemmas between opportunities, wages, travel time and cost of living. The objective of this paper is to analyze which municipal factors are associated with the highest rates of commuting in Brazilian municipalities in 2010 , understanding these movements as possibly spatially autocorrelated. For this purpose, techniques of spatial econometrics were used. Among the main findings is the need to consider spatial autocorrelation in the analysis of pendular mobility determinants. In addition, the results indicate that labor market and urban agglomeration variables are directly associated with greater inflows of commuters. On the other hand, the characteristics of urban infrastructure do not seem to explain commuting in the same way that it explains migratory attractiveness.
\end{abstract}

Keywords: labor market, commuting, spatial analysis.

JEL Code: J61, R10, C31. 


\section{INTRODUÇÃO}

A dissociação entre o município de residência e o de trabalho é um fenômeno crescente nos últimos anos (Ântico, 2005; Jardim, 2011; Moura, Branco, \& Firkowski, 2005; Moura, Delgado, \& Costa, 2013). Esse fenômeno, conhecido como pendularidade, está diretamente associado aos processos de ocupações desiguais no espaço, sendo, portanto, um movimento que reflete a expansão das atividades econômicas nos distintos territórios.

As teorias clássicas de localização espacial, ao investigar a configuração espacial das atividades econômicas, ajudam a compreender os movimentos pendulares. Tais estudos têm enfoque principal na análise matemática da localização das atividades, considerando distância e custo de transporte (Lösch, 1954; von Thünen, 1966). A concentração geográfica das atividades é resultado de forças de aglomeração (centrípetas), como o tamanho dos mercados, menores custos de transporte e maiores salários; e de dispersão (centrífugas), como a concorrência, congestionamentos e maiores custos de aluguéis (Krugman, 1998).

Os movimentos pendulares e seus determinantes têm sido amplamente estudados em diversos outros países, tornando-se um tema que ganha cada vez mais atenção nas discussões de Economia do Trabalho e Economia Regional e Urbana (Estados Unidos, Irlanda, Alemanha, Suécia, Canadá) (Mclafferty, 1997; Lloyd \& Shuttleworth, 2005; Papanikolaou, 2006; Sandow \& Westin, 2010; Axisa, Scott, \& Newbold, 2012).

Van Ommeren, Rietveld e Nijkamp (1997) examinam, usando dados em painel, como as informações imperfeitas do mercado e os custos de movimentação atuam sobre o comportamento pendular, em termos de distância do referido movimento, na Holanda, entre 1992 e 1993. Para capturar as imperfeições de mercado é observado: (i) se aqueles que recebem mais ofertas de emprego realizam menores deslocamentos pendulares, e (ii) se aqueles que mudaram recentemente de emprego realizam trajetos pendulares maiores. Os resultados corroboram a ambas hipóteses e apoiam a perspectiva de que os trabalhadores buscam por melhores alternativas de deslocamentos pendulares dentro do mercado de trabalho.

Os deslocamentos pendulares ajudam a compreender as deficiências existentes em termos de política habitacional, de transportes e mercado de trabalho nas distintas localidades. Papanikolaou (2006) mostra, através da análise de correlação para Alemanha no ano de 2003, a existência de uma conexão entre a densidade de empregos e a taxa de deslocamento pendular, de maneira que a diminuição da 
densidade de empregos eleva a taxa de deslocamento. Distintas provisões espaciais de bens públicos locais também podem, de alguma forma, afetar os movimentos pendulares intermunicipais (Nowotny, 2011).

Nesse sentido, a compreensão de como a provisão de bens públicos - tais como a presença de instituições de ensino ou centros de saúde - e as amenidades locais afetam os fluxos de deslocamento pendular intermunicipais, por motivo de trabalho, é investigada na Áustria por Nowotny (2011). Segundo o autor, partindo do pressuposto de que a provisão de bens públicos pode configurar um ganho adicional para a viagem pendular, influenciando positivamente tais fluxos, é razoável concluir que os indivíduos optam por trabalhar em um município com potencial de oferecer uma maior variedade de bens públicos ou amenidades do que seu local de residência.

Os indivíduos que vivem distantes das áreas centrais devem ser, em alguma medida, compensados pelos custosos deslocamentos pendulares. Essa compensação assume a forma de preços de habitação mais baixos, comparativamente com localizações mais próximas do CBD (Central Business District) (Muth, 1969; Mills, 1972; Brueckner, 1987).

Neste sentido, Dewey e Montes-Rojas (2009), em uma análise para os Estados Unidos em 2000, examinam os efeitos complementaridade e substituição entre distância do trabalho, comodidades regionais e preços de habitação, concluindo que a elasticidade dos salários em relação ao tamanho da cidade aumenta com a centralidade ocupacional. Os autores argumentam que, na medida em que o tamanho da cidade aumenta, os trabalhadores em ocupações mais centrais enfrentam situações menos desejáveis de preços de habitação e tempos de deslocamento, comparativamente com aqueles com empregos em áreas residenciais.

Alguns estudos analisam os fluxos pendulares nas distintas localidades do território brasileiro (Ântico, 2005; Jardim \& Ervatti, 2007, 2009; Miranda \& Domingues, 2008; Moura et al., 2005; Moura et al., 2013; Pereira \& Herrero, 2009). Contudo, a incorporação das técnicas de econometria espacial para a análise desses fluxos ainda não é devidamente explorada na literatura.

A percepção das variáveis de mobilidade populacional associadas aos aspectos de dependência espacial e às suas particularidades é algo que alguns estudos nacionais têm tratado. Nesse sentido, Mata, Oliveira, Pin e Resende (2007) analisam os principais determinantes da migração qualificada no Brasil, e os aspectos municipais que atraem tais migrantes. Através de modelos de econometria espacial, os autores notam que o maior dinamismo do mercado de trabalho, menor desi- 
gualdade renda, menor taxa de violência contra jovens, proximidade ao litoral e condições climáticas mais amenas são importantes condicionantes dos fluxos de migrantes qualificados.

Em uma análise para migração e migração de retorno intermunicipal no Brasil, Justo, Ferreira, Lima e Martins (2010) também incorporam as técnicas de econometria espacial para entender os determinantes desses movimentos. Os achados de Justo et al. (2010) apontam à relevância da renda esperada e de variáveis de infraestrutura urbana na determinação da migração, porém, estas mesmas variáveis não parecem muito importantes para migração de retorno. Logo, os autores argumentam que é possível a existência de processos motivacionais distintos que geram esses fluxos.

Justo e Silveira Neto (2008) também analisam os determinantes da migração interestadual no Brasil, porém, através de um painel espacial de dados dos censos demográficos de 1980, 1991 e 2000. Os autores percebem o importante papel da variável renda esperada para explicação da taxa líquida de migração, bem como outras variáveis, como aquelas associadas ao efeito da atratividade local.

Todos os estudos citados, que incorporam o processo de dependência espacial e que focam nos movimentos populacionais, têm dado a devida atenção à mudança de residência. Porém, as questões atreladas à localidade de trabalho - que estão, por sua vez, diretamente associadas aos deslocamentos casa-trabalho - tem recebido pouca atenção, no tocante a compreender esses movimentos como autocorrelacionados espacialmente.

Em relação à literatura sobre escolhas residenciais dos indivíduos, ressalta-se que as decisões de onde viver e trabalhar envolvem dilemas entre salários, tempo de deslocamento e custo de vida. Assim, a probabilidade individual de residência em uma determinada área é negativamente influenciada pelos níveis de custo de vida (preços de habitação) e positivamente afetada pelos níveis salariais. Ademais, os incentivos para que a população urbana realize o deslocamento pendular são maiores quanto maior o salário nos mercados vizinhos (So, Orazem, \& Otto, 2001).

Diante do exposto, o objetivo deste artigo é analisar aspectos importantes dos movimentos pendulares por motivo de trabalho nos municípios brasileiros de destino em 2010. Pretende-se investigar as características espaciais e os padrões dos fluxos pendulares. 
Com o intuito de compreender quais os fatores municipais associados com as maiores taxas de entrada de pendular (por motivo de trabalho); e, adicionalmente, traçar o perfil dos municípios que apresentam maiores taxas desse tipo de mobilidade, utilizaram-se técnicas de econometria espacial.

O estudo encontra-se dividido em seis seções, com esta introdução. $\mathrm{Na}$ próxima seção é explicada a metodologia empregada, a terceira seção abrange uma descrição detalhada dos dados utilizados. A quarta seção aborda uma caracterização dos municípios em relação aos movimentos pendulares. Finalmente, na quinta seção são analisados os determinantes dos movimentos pendulares e, na sequência, tem-se uma breve seção com as considerações finais.

\section{METODOLOGIA}

A estratégia empírica para análise do fluxo de deslocamento pendular, através das técnicas de econometria espacial, permite investigar os fatores municipais que afetam a taxa de deslocamento pendular, considerando a possível existência de autocorrelação espacial dessa variável.

A análise da taxa de deslocamento pendular para o município de trabalho é feita, a princípio, através da construção dos indicadores de correlação espacial global e local. Assim, foram realizados os testes I de Moran e LISA, objetivando examinar a existência de autocorrelação espacial nas taxas de entrada de pendulares.

Calculado tais indicadores para os municípios brasileiros, foi estimado um modelo que relaciona taxa de deslocamento pendular para o município de trabalho com aspectos de qualidade de vida, mercado de trabalho e distância das áreas centrais. A estimação do modelo básico é conduzida por meio do método de Mínimos Quadrados Ordinários (MQO). Para comprovar a existência de autocorrelação espacial no modelo, é realizado o teste I de Moran sobre os resíduos de MQO.

Na situação em que a presença de autocorrelação espacial é confirmada, deve ser verificado qual dos modelos espaciais mais se adequa ao problema de estudo. Os três modelos clássicos que ponderam a existência de autocorrelação espacial são: o modelo de defasagem espacial (ou SAR - Spatial Auto Regressive), o modelo de erro autorregressivo espacial (também conhecido como modelo de erro espacial ou SEM - Spatial Error Model) e o modelo de defasagem espacial com erro autorregressivo espacial ou SAC (Spatial Autoregressive Combined) (Almeida, 2012). 
No modelo de defasagem com erro espacial, a interação das regiões vizinhas ocorre tanto nas variáveis dependentes $(y)$, como do termo de erro $(\varepsilon)$. Formalmente, são incluídos no modelo uma defasagem espacial e um termo de erro dependente espacialmente (Keleijian \& Prucha, 1998). O modelo é representado por:

$$
\begin{gathered}
y=\rho W_{1} y+X \beta+\xi \\
\xi=\lambda W_{2} \xi+\varepsilon
\end{gathered}
$$

onde $\rho$ representa o coeficiente de defasagem espacial, $W$ é a matriz de pesos espaciais, sendo $W_{1} y$, o vetor de defasagem espacial da variável dependente e $W_{2} \xi$ a defasagem dos erros autocorrelacionados espacialmente. A forma reduzida revela que:

$$
y=\left(I_{n}-\rho W_{1}\right)^{-1} X \beta+\left(I_{n}-\rho W_{1}\right)^{-1}\left(I_{n}-\rho W_{2}\right)^{-1} \varepsilon
$$

O modelo SAR assume que a variável dependente de uma região depende do nível dessa variável nas regiões vizinhas. Esse modelo pode ser expresso por:

$$
\begin{gathered}
y=\rho W y+X \beta+\varepsilon \\
\varepsilon \sim N\left(0, \sigma^{2} I_{n}\right)
\end{gathered}
$$

Após algumas manipulações, a Equação 3 pode ser escrita em sua forma reduzida:

$$
y=\left(I_{n}-\rho W\right)^{-1} X \beta+\left(I_{n}-\rho W\right)^{-1} \varepsilon
$$

A Equação 4 informa que o modelo de defasagem espacial é especificado de maneira que a variável dependente, diga-se a taxa de deslocamento pendular por município de trabalho (taxa de entrada), é determinada pela média dos valores da variável dependente na vizinhança $(W y)$, pelas variáveis explicativas exógenas $(X)$ e por um termo de erro aleatório $(\varepsilon)$. O coeficiente autorregressivo espacial, $\rho$, capta os efeitos de transbordamento de $y$ sobre os vizinhos (Almeida, 2012). 
O modelo de erro espacial (SEM), por sua vez, capta e pondera a dependência espacial residual, de modo que o padrão espacial do termo de erro é constituído por efeitos não modelados e não correlacionados com as variáveis explicativas da regressão (Almeida, 2012). Assim, o termo de erro da região $i$ está correlacionado com o erro da região $j$. No modelo de erro espacial, modela-se o erro do modelo de regressão linear da seguinte maneira:

$$
\begin{gathered}
y=X \beta+\xi \\
\xi=\lambda W \xi+\varepsilon
\end{gathered}
$$

Através de manipulação da Equação (5), obtém-se a expressão reduzida do modelo a ser estimado:

$$
\begin{gathered}
y=X \beta+\left(I_{n}-\lambda W\right)^{-1} \varepsilon \\
\varepsilon \sim N\left(0, \sigma^{2} I_{n}\right)
\end{gathered}
$$

Tal que o parâmetro do erro autorregressivo espacial é dado por $\lambda$ e $W \xi$ é um vetor $n$ por 1 de defasagem em $\xi$.

Segundo Florax, Folmer e Rey (2003), os seguintes passos devem ser seguidos para especificação do modelo espacial: primeiro, estima-se o modelo através do método de MQO; o segundo passo é testar a hipótese de ausência de dependência espacial - tanto devido à omissão da defasagem espacial da variável dependente (SAR), como devido à omissão do erro espacial autorregressivo (SEM) -, usando $M L_{\rho}$ e $M L_{\lambda}$, respectivamente. Caso ambos os testes não sejam estatisticamente significativos, a estimação de MQO é utilizada como a regressão final. Caso contrário, o modelo escolhido deverá ser aquele que apresenta a estatística do teste de ML mais significativa; e quando $M L_{\rho}$ for estatisticamente significativo, porém $M L_{\lambda}$ não, deve-se estimar o modelo SAR, caso contrário, estima-se o modelo SEM.

Finalmente, através desse procedimento proposto por Florax et al. (2003), foi selecionado o modelo econométrico adequado para analisar a relação existente entre as taxas de deslocamento pendular para as localidades de trabalho e as variáveis atreladas ao mercado de trabalho, amenidades locais e distância desses territórios.

O método de estimação de MQO pode não ser apropriado para os modelos de econometria espacial. Para o modelo SAR, o coeficiente espacial $\rho$ é viesado e inconsistente se estimado por MQO; e, para o modelo SEM, as estimativas de MQO são ineficientes, apesar de não viesadas e consistentes. Assim, quando a 
normalidade não é garantida, é possível estimar os modelos SAR e SEM usando o método de Máxima Verossimilhança (Kelejian \& Prucha, 1999; Almeida, 2012).

\section{DADOS}

Os dados utilizados para a análise do deslocamento pendular nos municípios brasileiros em 2010 são provenientes de vários bancos de dados. A descrição detalhada de cada variável está na Tabela 1, a seguir. Utiliza-se a divisão territorial municipal de 5564 municípios em 2010 como unidade de análise ${ }^{1}$.

A taxa de deslocamento pendular da presente análise considera aqueles indivíduos que cruzam as fronteiras territoriais municipais para trabalhar ${ }^{2}$. Assim, o percentual de deslocamento pendular da força de trabalho para cada um dos 5564 municípios brasileiros em 2010 - que é a variável dependente a ser analisada - é obtido da razão entre o volume do fluxo pendular para o município $i$ e o volume total da mobilidade pendular no Brasil. É importante ficar evidente que, ao optar-se por analisar o volume do fluxo pendular pela perspectiva da "entrada", isto é, o fluxo por município de trabalho, examinam-se, efetivamente, quais são as características municipais associadas às maiores taxas de entrada desse tipo de trabalhador, o que permite visualizar melhor os possíveis clusters de elevada taxa de entrada de mão de obra pendular, bem como os aspectos desses municípios receptores.

Tabela 1. Descrição das variáveis

\begin{tabular}{lll}
\hline Variável & Definição & Fonte \\
\hline $\begin{array}{l}\text { Taxa de entrada } \\
\text { pendular (\%) }\end{array}$ & $\begin{array}{l}\text { Mede a proporção de indivíduos pendulares } \\
\text { que estão entrando no município para tra- } \\
\text { balhar, do total de pendulares do Brasil em } \\
2010 .\end{array}$ & $\begin{array}{l}\text { Censo } \\
\text { Demográfico } \\
\text { de } 2010\end{array}$ \\
\hline $\begin{array}{l}\text { Distância até a capital } \\
(\mathrm{km})\end{array}$ & $\begin{array}{l}\text { Distância à capital estadual em km para os muni- } \\
\text { cípios da divisão político administrativa vigente. }\end{array}$ & IPEADATA \\
& &
\end{tabular}

1 O município de Nazária (PI) é excluído da análise, devido à ausência de dados para essa localidade no ano de 2010.

2 Neste sentido, foram considerados pendulares aqueles indivíduos que afirmaram trabalhar em um município distinto daquele de residência, da amostra de trabalhadores (aqueles que informaram ocupação e renda do trabalho não nula) entre 18 e 65 anos de idade. 
$\begin{array}{ll}\text { Densidade populacional } & \begin{array}{l}\text { Razão entre a população residente do mu- } \\ \text { nicípio e sua área, medida em quilômetros } \\ \text { (hab. por } \mathrm{km}^{2} \text { ) }\end{array} \\ \text { quadrados. }\end{array}$

Medido a partir do indicador "renda per capita", através da fórmula: [ln (valor obserIDHM - renda $\quad$ vado do indicador) - ln (valor mínimo)] / [ln (valor máximo) - ln (valor mínimo)], onde PNUD os valores mínimo e máximo são R\$ 8.00 e $\mathrm{R} \$ 4033.00$ (a preços de agosto de 2010).

Média ponderada de seis indicadores, são eles: atendimento à educação infantil; abandono no ensino fundamental; distorção idade-série no ensino fundamental; docentes com ensino superior no ensino fundamental; média de

IFDM - educação horas-aula diárias no ensino fundamental; e FIRJAN resultado do IDEB no ensino fundamental. Os indicadores são extraídos do Censo Escolar e do Índice de Desenvolvimento da Educação Básica (IDEB), do Ministério da Educação (MEC). O índice varia entre 0 e 1.

Média ponderada de quatro indicadores, são eles: proporção de atendimento adequado de pré-natal; óbitos por causas mal definidas; óbitos infantis por causas evitáveis; interIFDM - saúde nação sensível à atenção básica (ISAB). Os indicadores são extraídos do Sistema de Informações sobre Mortalidade (SIM) e do Sistema de Informações sobre Nascidos Vivos (SINASC). O índice varia entre 0 e 1.

Mede a desigualdade na distribuição de indivíduos de 18 anos de idade ou mais, ocupados

Índice de Theil-L dos rendimentos do trabalho segundo o rendimento de todos os trabalhos, excluídos aqueles sem rendimento do trabalho. É nulo quando não existir desigualdade PNUD de renda entre eles e tende ao infinito quando a desigualdade tender ao máximo.

Fonte: elaboração própria.

Nota: as variáveis densidade populacional e distância até a capital são utilizadas em logaritmo natural nos modelos econométricos. 
Os dados referentes às variáveis explicativas do modelo de regressão buscam englobar os principais fatores que possam estar diretamente ou indiretamente associados ao percentual de mão de obra pendular entrante nos distintos municípios. Espera-se que as variáveis de mercado de trabalho estejam fortemente associadas a esse tipo de deslocamento, pelo fato de o propósito final ser exatamente o "trabalho". Contudo, é possível que haja distintas justificativas para o trabalhador optar por trabalhar em um munícipio e residir em outro. Então, as características geográficas e de distintas provisões de bens públicos também podem influenciar esses fluxos pendulares.

A ideia que prevalece é a de que o indivíduo pondera as características das localidades de residência e de trabalho, quando toma a decisão pela pendularidade (Ântico, 2005; Moura et al., 2005; Moura et al., 2013). Logo, os fluxos pendulares estão interligados às especificidades municipais.

Utiliza-se, como variável de mercado de trabalho, o índice de Theil-L de desigualdade nos rendimentos do trabalho. Espera-se que a desigualdade no rendimento do trabalho assuma uma relação negativa com a taxa de deslocamento pendular por município de trabalho (entrada). Contudo, é preciso cuidado ao se fazer esse tipo de generalização, uma vez que é possível que uma maior desigualdade de rendimento do trabalho esteja associada a uma maior probabilidade ou interesse de inserção no mercado de trabalho, tanto para indivíduos no topo da distribuição de capital humano (com alta qualificação), como para aqueles que estão na base dessa distribuição.

As variáveis que tentam captar amenidades urbanas são: densidade populacional, padrão de vida (IDHM - renda) e o IFDM nas suas dimensões saúde e educação. O IDHM e ambos IFDM, educação e saúde, variam de 0 a 1. Quanto mais próximo de 1, maior o desenvolvimento humano municipal na referida dimensão. Supondo que os indivíduos percebem mais oportunidades de emprego em localidades mais centrais e desenvolvidas, é possível que as taxas de entrada pendular sejam maiores em territórios com melhores indicadores de amenidades urbanas. Contudo, é extremamente importante perceber que o movimento pendular dissocia-se, em certa medida, do movimento migratório nesse aspecto da relevância que é dada para a qualidade de vida no município de destino, uma vez que, para o pendular, o município de destino pode ser unicamente o seu local de trabalho, e não o ambiente que ele escolhe para viver, como ocorre com o migrante. Diante do exposto, o efeito dessas variáveis sobre o fluxo pendular pode ser ambíguo. 
Assume-se a distância em quilômetros até a capital estadual como proxy para acessibilidade/custos de transporte até as áreas centrais. A hipótese clássica subjacente a essa questão da acessibilidade e aos ganhos de transbordamento da proximidade das áreas centrais é a de que localidades mais afastadas do território metropolitano apresentam menores fluxos de entrada de trabalhadores pendulares (Muth, 1969; Mills, 1972; Brueckner, 1987). Porém, essa ainda é uma questão a ser analisada para o Brasil. As estatísticas descritivas para todas as variáveis podem ser observadas na Tabela A1, no Apêndice 3 .

\section{CARACTERÍSTICAS DOS MUNICÍPIOS BRASILEIROS EM RELAÇÃO À PENDULARIDADE}

Com o objetivo de analisar quais localidades têm apresentado um processo mais intenso de entrada de indivíduos para atividades laborais, o Censo Demográfico de 2010 permite extrair as três variáveis-chave dessa análise: volume de entrada de pendulares, volume de saída de pendulares, e o total da população.

Tabela 2. Volume dos deslocamentos pendulares nos estados do Brasil em 2010

\begin{tabular}{|c|c|c|c|}
\hline Ranking nacional & UF & $\begin{array}{c}\text { Volume dos deslocamentos } \\
\text { pendulares (entradas) }\end{array}$ & $\%$ \\
\hline 1 & SP & 2663596 & 31.25 \\
\hline 2 & RJ & 1097858 & 12.88 \\
\hline 3 & $\mathrm{MG}$ & 824078 & 9.67 \\
\hline 4 & $\mathrm{RS}$ & 554356 & 6.50 \\
\hline 5 & PR & 494400 & 5.80 \\
\hline 6 & $\mathrm{PE}$ & 471580 & 5.53 \\
\hline 7 & $\mathrm{SC}$ & 310862 & 3.65 \\
\hline 8 & $\mathrm{BA}$ & 305441 & 3.58 \\
\hline 9 & $\mathrm{GO}$ & 241950 & 2.84 \\
\hline 10 & ES & 232459 & 2.73 \\
\hline 11 & $\mathrm{CE}$ & 200544 & 2.35 \\
\hline
\end{tabular}

3 A seleção dessas variáveis explicativas baseou-se naquelas utilizadas em modelos de análise de migração e nos argumentos teóricos relativos a pendularidade. 


\begin{tabular}{|c|c|c|c|}
\hline 12 & $\mathrm{DF}$ & 174324 & 2.05 \\
\hline 13 & PA & 165297 & 1.94 \\
\hline 14 & $\mathrm{RN}$ & 138509 & 1.63 \\
\hline 15 & PB & 113910 & 1.34 \\
\hline 16 & MA & 105229 & 1.23 \\
\hline 17 & SE & 99851 & 1.17 \\
\hline 18 & MT & 82618 & 0.97 \\
\hline 19 & $\mathrm{AL}$ & 65576 & 0.77 \\
\hline 20 & PI & 53746 & 0.63 \\
\hline 21 & MS & 46545 & 0.55 \\
\hline 22 & TO & 21881 & 0.26 \\
\hline 23 & RO & 21380 & 0.25 \\
\hline 24 & $\mathrm{AM}$ & 16995 & 0.20 \\
\hline 25 & $\mathrm{AP}$ & 11425 & 0.13 \\
\hline 26 & $\mathrm{AC}$ & 5294 & 0.06 \\
\hline \multirow[t]{2}{*}{27} & RR & 3714 & 0.04 \\
\hline & Brasil & 8523418 & 100 \\
\hline
\end{tabular}

Fonte: Elaboração própria a partir dos microdados do Censo Demográfico de 2010.

Notas: O volume dos deslocamentos pendulares é composto do total de indivíduos que afirmam trabalhar em um município distinto daquele de residência, logo observa-se qual é exatamente o destino de trabalho desse indivíduo pendular, e não a localidade de residência.

A Tabela 2 mostra o volume dos deslocamentos pendulares nos estados brasileiros, especificamente o fluxo de entrada. Esse volume refere-se ao montante de indivíduos que afirmaram trabalhar em um município da UF distinto daquele de residência. Como esperado, os cinco estados com maiores taxas de deslocamento pendular são aqueles pertencentes às regiões Sul e Sudeste, que configuram economias mais desenvolvidas e com grande dinamismo produtivo.

Como é observado o deslocamento exclusivamente por motivo de trabalho, obviamente o volume desses movimentos está associado ao dinamismo econômico das diferentes regiões e estados brasileiros. É visível que todos os estados do Norte, com exceção do Pará, estão entre aqueles com menores volumes de deslocamentos 
pendulares. Além de uma dinâmica econômica particular, comparativamente ao resto do país, os municípios pertencentes aos estados de Roraima, Amazonas, Acre, Rondônia, Tocantins e Amapá estão entre os que apresentam, em média, menor densidade populacional.

Na Tabela 3 é possível observar os dez municípios mais bem classificados em termos nacionais para o indicador de pendularidade. Nota-se claramente que os principais municípios receptores de mão de obra pendular são exatamente as capitais estaduais, evidenciando a relevância das áreas metropolitanas como principal foco de destino dos trabalhadores pendulares.

Tabela 3. Os dez municípios mais bem classificados em termos nacionais para o indicador de pendularidade em 2010

\begin{tabular}{cccc}
\hline $\begin{array}{l}\text { Ranking } \\
\text { nacional }\end{array}$ & Município & $\begin{array}{l}\text { Entrada de } \\
\text { pendulares }\end{array}$ & $\%$ \\
\hline 1 & São Paulo (SP) & 832968 & 9.85 \\
\hline 2 & Rio de Janeiro (RJ) & 589586 & 6.97 \\
\hline 3 & Belo Horizonte (MG) & 322379 & 3.81 \\
\hline 4 & Recife (PE) & 251602 & 2.97 \\
\hline 5 & Porto Alegre (RS) & 212977 & 2.52 \\
\hline 6 & Curitiba (PR) & 196309 & 2.32 \\
\hline 7 & Brasília (DF) & 174324 & 2.06 \\
\hline 8 & Goiânia (GO) & 141504 & 1.67 \\
\hline 9 & Niterói (RJ) & 122253 & 1.44 \\
\hline 10 & Vitória (ES) & 114032 & 1.35 \\
\hline
\end{tabular}

Fonte: elaboração própria a partir dos microdados do Censo Demográfico de 2010.

A respeito daqueles municípios que apresentaram as menores taxas de entrada de trabalhadores pendulares, em relação ao total nacional, destacam-se localidades mais afastadas de suas respectivas capitais estaduais, tais como Montauri (230 Km) e Vanini (246 Km), ambas no Rio Grande do Sul; Virgolândia (311 Km), Presidente Kubitschek (298 Km), Cipotânea (175 Km), em Minas Gerais; Formosa do Sul (626 Km), em Santa Catarina e Ibiquera (386 Km), na Bahia. Estes municípios citados, não por acaso, estão entre as 10 localidades com menores taxas de entrada da força de trabalho pendular. Vale ressaltar que seis municípios não 
apresentaram algum fluxo pendular de "entrada" em 2010, a citar Santa Isabel do Rio Negro (AM), Tamboril do Piauí (PI), Nicolau Vergueiro (RS), Porto Walter (AC), São João da Serra (PI) e Paranhos (MS).

Estes resultados evidenciam que o fator distância dos municípios das áreas centrais de seus respectivos estados está de certa forma associado aos fluxos pendulares que os municípios receberam em 2010. Essa realidade é facilmente entendida teoricamente à luz dos modelos de equilíbrio urbano de Muth (1969) e Mills (1972), que consideram o efeito da acessibilidade ao CBD (Central Business District) sobre a localização dos domicílios - isto é, o consumo de habitação - e a determinação do local de trabalho.

\section{DETERMINANTES DOS FLUXOS PENDULARES}

O objetivo dessa seção é analisar quais os fatores que explicam a taxa de deslocamento pendular, por município de trabalho, no Brasil. Isto é, busca-se entender quais as características municipais que importam para que o município exerça uma atratividade da mão de obra pendular.

A Figura 1 mostra o percentual de indivíduos pendulares, do total de pendulares do Brasil em 2010, que estão entrando no município para trabalhar. A análise do mapa indica que há uma certa concentração de municípios que obtiveram maiores indicadores de pendularidade.

Os maiores recebedores de trabalhadores de outros municípios estão localizados em grande parte nos estados de São Paulo e Rio de Janeiro, e nas regiões próximas às capitais. No Nordeste, as maiores concentrações estão nas regiões metropolitanas de Recife e Salvador. Ainda nesse contexto, destacam-se os municípios de Niterói, no Rio de Janeiro, e Campinas e São Bernardo do Campo, em São Paulo. Esses três municípios, de forma individual, receberam mais de $1 \%$ do total de pendulares no Brasil, à frente de capitais como Salvador e Florianópolis. A justificativa para tal reside no fato de que esses municípios estão inseridos em um ambiente econômico bastante dinâmico. O município de Niterói é o segundo maior empregador do estado do Rio de Janeiro, tendo a indústria do petróleo como setor fundamental. Já Campinas e São Bernardo do Campo se destacam pelo seu parque metalúrgico e automotivo, respectivamente, setores que demandam uma grande quantidade de trabalho. 
Figura 1. Proporção de indivíduos pendulares que estão entrando no município para trabalhar do total de pendulares do Brasil em 2010

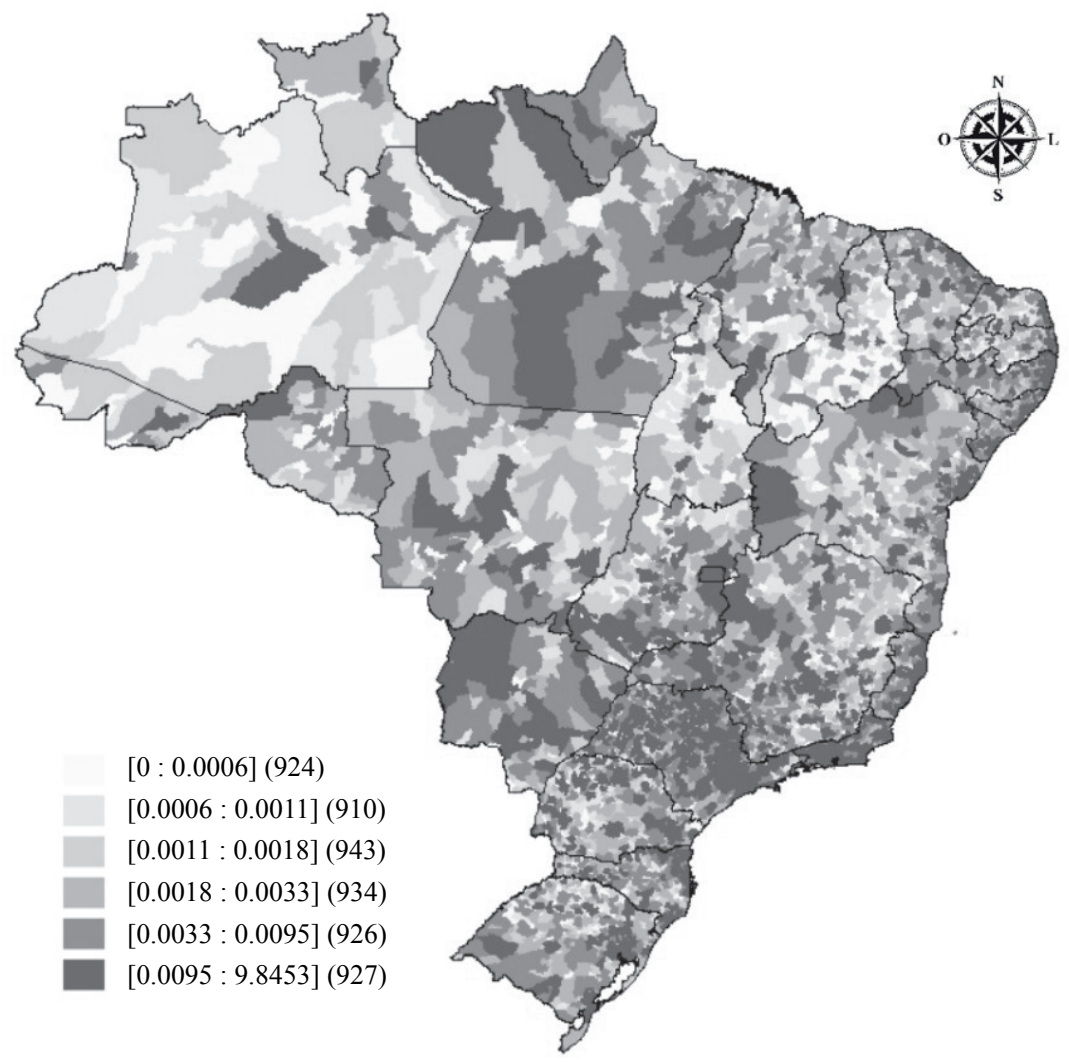

Fonte: elaboração própria.

Para examinar a possível existência de autocorrelação espacial no indicador de pendularidade, foi implementado o teste I de Moran. Os valores da estatística variam entre 1 e -1 . Um resultado próximo a 1 indica uma autocorrelação positiva, nesse caso, há uma similaridade entre os valores do atributo e sua localização no espaço. Já a autocorrelação espacial negativa (valor próximo a -1) indica a existência de uma dissimilaridade entre os valores do atributo e sua localização espacial. Nesse caso, altos (baixos) valores de uma variável $(y)$ tendem a estar próximos a baixos (altos) da mesma variável em regiões vizinhas (Wy) (Almeida, 2012). 
A seleção da matriz de dependência espacial baseou-se em um argumento teórico a respeito da pendularidade. Para a construção da matriz $W$ foi considerado que o efeito da pendularidade de um município sobre os vizinhos se dissipa com a distância. Isto é, quanto maior a distância entre os municípios, menor será a interação entre eles e, portanto, menor será o peso atribuído. De maneira análoga, municípios mais próximos entre si recebem pesos maiores ${ }^{4}$. A principal razão para esta escolha é o fato de que a decisão pela pendularidade é diretamente influenciada pela distância entre o município de residência e aquele ao qual se pretende trabalhar (Muth, 1969; Mills, 1972; Moura et al., 2013). Portanto, optou-se pela matriz de distância inversa.

A proporção de indivíduos pendulares que estão entrando no município para trabalhar (Figura 1) apresentou I de Moran de 0.133 (valor-p de 0.002). Segundo o sinal da estatística, há evidência de autocorrelação espacial positiva para o indicador de pendularidade. Isto implica que, municípios que apresentam maiores (menores) percentuais de indivíduos pendulares, são vizinhos de municípios que também apresentam maior (menor) pendularidade. Outra estatística utilizada foi o indicador local de associação espacial (LISA), também conhecido como I de Moran local. A estatística LISA é capaz de capturar padrões locais de autocorrelação espacial, possibilitando a detecção da existência de clusters, dados os valores de uma determinada variável no espaço. Os municípios apresentados na Figura 2 são aqueles que passaram no teste de significância estatística de 5\% do I de Moran Local. Vale notar que os municípios caracterizados como sem vizinhos são aqueles em que a sua própria área geográfica excede o raio de $60 \mathrm{~km}$ em torno do seu centroide, conforme definido na matriz de distância inversa.

A análise da Figura 2 permite a identificação da existência de uma concentração de clusters que seguem o padrão alto-alto, localizados principalmente nas grandes regiões metropolitanas do Brasil e em seus entornos. O maior aglomerado está localizado no estado de São Paulo. Há uma grande concentração de municípios que apresentaram alto indicador de pendularidade em uma região que se estende desde o litoral, na mesorregião metropolitana de Santos, até o município de Cordeirópolis, na mesorregião de Piracicaba, distante $160 \mathrm{~km}$ da capital do estado. A pendularidade é bastante intensa, em especial, na Mesorregião Metropolitana de São Paulo. Dos 45 municípios que formam a mesorregião, 37 foram identificados, segundo a estatística LISA, como municípios com alta pendularidade, circundados

4 Assume-se, como distância de corte, um raio de $60 \mathrm{~km}$ em torno dos municípios, uma vez que as distâncias médias dos deslocamentos pendulares com destino às principais metrópoles brasileiras não excedem 60 km (Lobo, 2016). 
por vizinhos que também apresentaram um elevado indicador de pendularidade. Cunha, Stoco, Dota, Negreiros e Miranda (2013) já haviam identificado a desconcentração produtiva e suas consequências socioespaciais (como é o caso das novas formas de urbanização e localização da população) como sendo alguns dos possíveis condicionantes desse processo na Mesorregião Metropolitana Paulista.

Figura 2. Proporção de indivíduos pendulares que estão entrando no município para trabalhar, do total de pendulares do Brasil em 2010

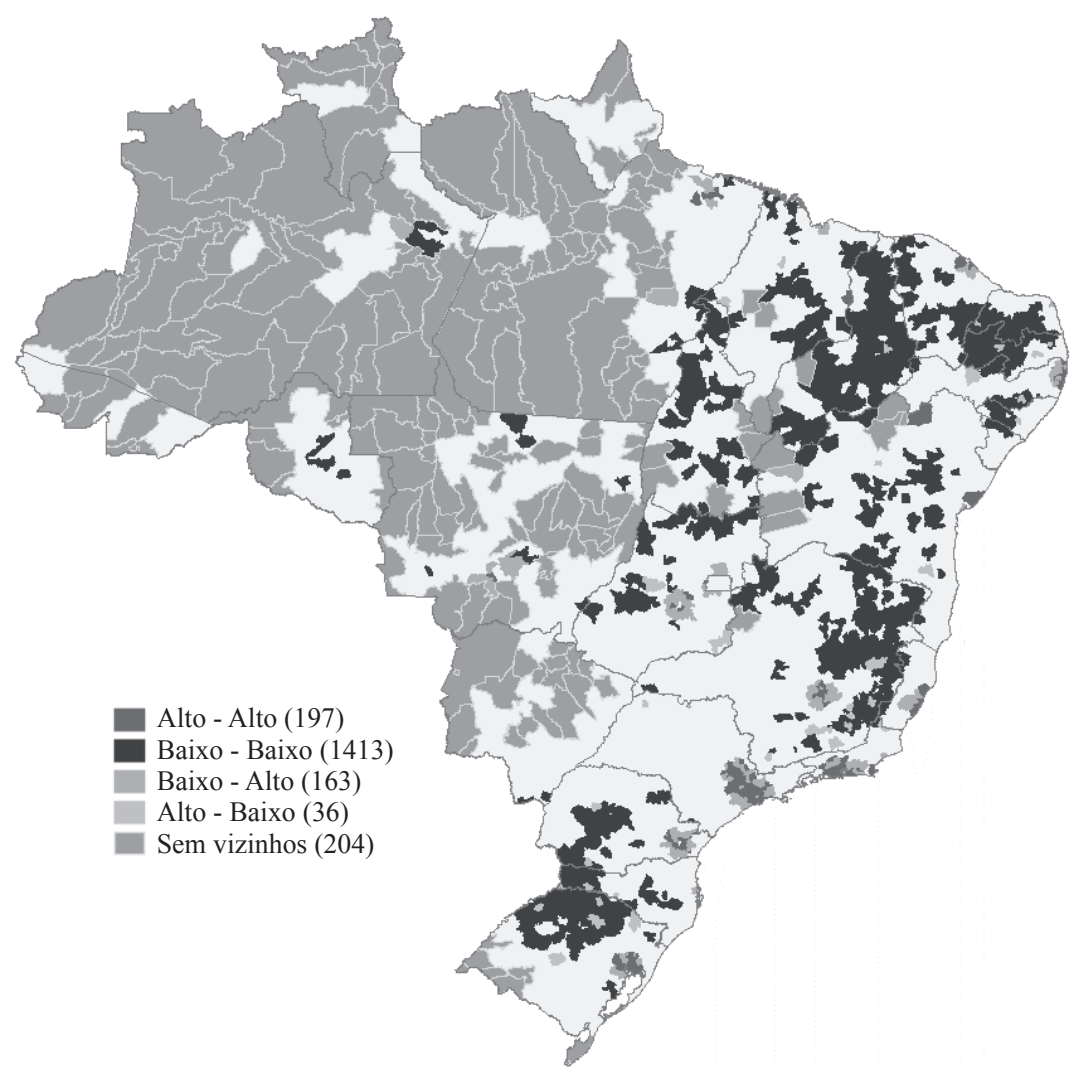

Fonte: elaboração própria. 
O cluster observado no estado do Rio de Janeiro também merece destaque. Apenas a Mesorregião Metropolitana do Rio de Janeiro concentra aproximadamente $83 \%$ dos municípios que fazem parte do aglomerado alto-alto.

É importante notar também a existência de diversos pequenos clusters de padrão alto-alto em territórios próximos do litoral - grande parte das capitais brasileiras encontram-se no litoral -, caracterizando um fenômeno tradicional de atração pendular para as capitais.

Tabela 4. Determinantes dos fluxos pendulares no Brasil: Estimação dos modelos econométricos

\begin{tabular}{|c|c|c|c|}
\hline \multirow[t]{2}{*}{$\begin{array}{l}\text { Variável dependente: } \\
\text { Taxa de entrada pendular }\end{array}$} & MQO & $\begin{array}{c}\text { Modelo com Lag } \\
\text { Espacial } \\
(\mathrm{SAR}-\mathrm{MV})\end{array}$ & $\begin{array}{c}\text { Modelo com Erro } \\
\text { Espacial } \\
(\mathrm{SEM}-\mathrm{MV})\end{array}$ \\
\hline & $(1)$ & (2) & (3) \\
\hline \multirow[t]{2}{*}{ Constante } & 0.013 & 0.010 & $0.151 * * *$ \\
\hline & $(0.029)$ & $(0.030)$ & $(0.037)$ \\
\hline \multirow[t]{2}{*}{ Distância até a capital (log) } & $-0.055^{* * *}$ & $-0.054 * * *$ & $-0.081 * * *$ \\
\hline & $(0.003)$ & $(0.004)$ & $(0.004)$ \\
\hline \multirow[t]{2}{*}{ Densidade populacional (log) } & $0.012 * * *$ & $0.012 * * *$ & $0.007 * * *$ \\
\hline & $(0.002)$ & $(0.002)$ & $(0.002)$ \\
\hline \multirow[t]{2}{*}{ IDHM - renda } & $0.367 * * *$ & $0.362 * * *$ & $0.390 * * *$ \\
\hline & $(0.044)$ & $(0.044)$ & $(0.052)$ \\
\hline \multirow[t]{2}{*}{ IFDM - educação } & 0.008 & 0.006 & -0.005 \\
\hline & $(0.028)$ & $(0.028)$ & $(0.031)$ \\
\hline \multirow[t]{2}{*}{ IFDM - saúde } & $-0.069 * * *$ & $-0.068 * * *$ & $-0.051 * *$ \\
\hline & $(0.020)$ & $(0.020)$ & $(0.021)$ \\
\hline \multirow[t]{2}{*}{$\begin{array}{l}\text { Theil-L dos rendimentos do } \\
\text { trabalho }\end{array}$} & $0.162 * * *$ & $0.164 * * *$ & $0.155^{* * *}$ \\
\hline & $(0.021)$ & $(0.021)$ & $(0.022)$ \\
\hline
\end{tabular}




\begin{tabular}{|c|c|c|c|}
\hline \multirow[t]{2}{*}{ W_TMIGPEND } & \multicolumn{3}{|c|}{$0.059 * *$} \\
\hline & \multicolumn{3}{|c|}{$(0.029)$} \\
\hline \multirow[t]{2}{*}{$\lambda$} & & & $0.350 * * *$ \\
\hline & & & $(0.025)$ \\
\hline $\mathrm{R}^{2}$ & 0.116 & 0.117 & 0.139 \\
\hline $\mathrm{R}^{2}$ Ajustado & 0.116 & & \\
\hline Log-Likelihood & & 1592 & 1627 \\
\hline AIC & & -3168 & -3241 \\
\hline Observações & 5564 & 5564 & 5564 \\
\hline
\end{tabular}

Fonte: elaboração própria. Obs.: Matriz de inversa da distância (raio de 60 km).

Notas: desvios-padrão entre parênteses. ${ }^{* * *}$ Estatisticamente significante a $1 \%$. **Estatisticamente significante a $5 \%$. *Estatisticamente significante a $10 \%$.

A Tabela 4, acima, apresenta os resultados dos distintos modelos de análise dos determinantes da mobilidade pendular nas cidades brasileiras. Na primeira coluna, é observado o modelo estimado por MQO. A existência de autocorrelação espacial, confirmada na Tabela 5 de diagnóstico para dependência espacial, pode violar os pressupostos de erros homoscedástico e não autocorrelacionado do modelo de regressão clássico, e, por conseguinte, as estimativas dos parâmetros do modelo de MQO podem gerar resultados inconsistentes e/ou ineficientes ${ }^{5}$.

Assim, para contornar tais problemas, os dois modelos clássicos de econometria espacial que ponderam a existência de autocorrelação espacial, SAR e SEM, são estimados e testados para ver qual deles melhor se adequa à estrutura dos dados e ao problema espacial (Anselin, 1988). Seguindo a estratégia de Florax et al. (2003), o modelo escolhido deverá ser aquele que apresenta a estatística do

5 No modelo de defasagem espacial, a presença de autocorrelação espacial não tratada torna os coeficientes de MQO não consistentes; e no modelo de erro espacial, a presença de autocorrelação espacial não tratada ocasiona a perda de eficiência dos parâmetros estimados (Almeida, 2012). Logo, se o modelo for incorretamente estimado por MQO, o estimador dos parâmetros não é eficiente e os erros padrão de MQO são inconsistentes, consequentemente, toda a inferência habitual deixa de ser válida. 
teste de ML mais significativa ${ }^{6}$. Assim, como apenas $M L_{\lambda}$ é significativo, analisa-se o modelo SEM, expresso na Equação (6). Os resultados para esse modelo são apresentados na terceira coluna da Tabela 4. Para fins apenas de comparação, na segunda coluna dessa tabela são ilustrados os resultados do modelo SAR. Os modelos com correção espacial são estimados pelo método de Máxima Verossimilhança $(\mathrm{MV})^{7}$.

Tabela 5. Diagnóstico para dependência espacial

\begin{tabular}{lcc}
\hline Teste & Valor & Prob. \\
\hline I de Moran (resíduos) & 0.038 & 0.000 \\
\hdashline Multiplicador de Lagrange (defasagem) & 1.998 & 0.157 \\
\hdashline ML robusto (defasagem) & 214.665 & 0.000 \\
\hline Multiplicador de Lagrange (erro) & 37.674 & 0.000 \\
\hline ML robusto (erro) & 250.341 & 0.000 \\
\hline
\end{tabular}

Fonte: elaboração própria. Obs.: matriz de inversa da distância (raio de 60 km).

Observando os resultados do modelo SEM, fica evidente a importância das variáveis associadas ao mercado de trabalho -isto é, IDHM-renda e desigualdade no rendimento do trabalho - na determinação dos fluxos pendulares, corroborando aos argumentos teóricos que já haviam sido mencionados, de que os fluxos pendulares estão especialmente interligados às características espaciais do mercado de trabalho.

O resultado para o indicador de desigualdade no rendimento do trabalho carece de uma investigação particular. Para os indivíduos mais qualificados, o deslocamento pendular passa a compensar diante da possibilidade do ganho salarial mais elevado (característico de localidades com maior desigualdade de renda do

6 O procedimento clássico de Florax, Folmer e Rey (2003) consiste, primeiramente, na estimação do modelo de regressão linear por MQO. Em seguida, computam-se $M L_{\lambda}$ e $M L_{\rho}$. Caso $M L_{\lambda}>M L_{\rho}$ ou $M L_{\lambda}$ for significativo e $M L_{\rho}$ não, é estimado o modelo SEM. Por outro lado, se $M L_{\rho}>M L_{\lambda}$ ou $M L_{\rho}$ for significativo e $M L_{\lambda}$ não, estima-se o modelo SAR.

7 A estimação também é conduzida por meio do Método dos Momentos Generalizados (GMM) e apresentada no Apêndice, na Tabela A2. 
trabalho). De maneira similar, para os indivíduos menos qualificados, se deslocar para localidades com maior desigualdade de renda do trabalho pode ser a única maneira dele se inserir no mercado de trabalho. Claramente, no primeiro caso tem-se um deslocamento pendular que ocorre por interesse individual (de obtenção de um ganho salarial maior), e, no segundo caso, um deslocamento que ocorre por necessidade. Independentemente da motivação individual, é razoável que, no agregado, o fluxo de deslocamento pendular seja maior para localidades com maior desigualdade salarial.

Outro aspecto importante é que a variável de distância até a capital estadual revela que localidades mais afastadas apresentam estatisticamente menores taxas de entrada de pendulares. Isto significa que os municípios mais afastados da capital possuem uma força atrativa de trabalhadores pendulares relativamente menor. Adicionalmente, a densidade populacional indica que municípios mais povoados exibem maior fluxo de entrada de pendulares, o que pode estar associado à hipótese de externalidades da aglomeração urbana.

As variáveis associadas à qualidade de vida no município de trabalho, ou seja, IFDM nas dimensões saúde e educação, apresentam efeitos distintos do esperado. O IFDM-educação não afeta de maneira significativa a taxa de deslocamento pendular de entrada. O fato curioso foi que o IFDM-saúde se mostrou significativo e apresentou uma relação negativa com a pendularidade, em todos os modelos. Isto pode estar ocorrendo por essa variável carregar outros aspectos municipais, o que dificulta a sua análise. Compreendendo melhor tal indicador, como este se refere à existência de uma rede de saúde adequada no município, é possível que um IFDM-saúde elevado esteja relacionado a uma população mais saudável e com melhores indicadores de longevidade, o que, indiretamente, pode caracterizar um mercado de trabalho local mais concorrido.

O coeficiente $\lambda$ do erro autorregressivo mostra-se significativo a $1 \%$ e positivo, sinalizando que um choque positivo na economia de um município vizinho se propaga em um benefício para o município em análise. No modelo de defasagem espacial, o termo $\rho$ é o coeficiente da defasagem espacial da variável dependente. Seu valor positivo é esperado, visto que municípios em que a entrada de trabalhadores é elevada são, em geral, vizinhos de municípios com elevada entrada de pendulares. Este resultado já havia sido indicado no exame da estatística LISA (Figura 2). 


\section{CONSIDERAÇÕES FINAIS}

O trabalho analisou quais características municipais são determinantes para a atração da mão de obra pendular, com enfoque nos aspectos de mercado de trabalho, amenidades urbanas e distância das áreas centrais. A identificação do indivíduo pendular engloba as pessoas que afirmaram trabalhar em um município distinto daquele de moradia, em 2010. O estudo dos fluxos de deslocamentos pendulares é de extrema relevância quando pode contribuir para a compreensão do processo de estruturação dos municípios.

Os testes apontaram para a necessidade da ponderação da autocorrelação espacial nos modelos econométricos de análise dos determinantes da mobilidade pendular intermunicipal. Assim, foram estimados modelos econométricos espaciais que captam tais efeitos. Os resultados desses modelos indicam a importância das variáveis de mercado de trabalho influenciando na taxa de entrada pendular, nos municípios brasileiros. Outro achado interessante foi a comprovação de que quanto maior a distância do município de análise em relação à capital, menores são os fluxos pendulares em direção a esse município.

Apesar dos movimentos migratórios (de mudança de residência) serem amplamente estudados, nota-se que ainda há importantes lacunas atreladas à dissociação entre o município de moradia e o de trabalho a serem exploradas. O presente artigo evidencia como os deslocamentos casa-trabalho parecem distintos dos movimentos migratórios, do ponto de vista motivacional, o que ilustra a importância de que os fluxos pendulares sejam analisados levando-se em consideração suas peculiaridades. Adicionalmente, destaca-se a necessidade de que políticas públicas, especialmente aquelas voltadas para o mercado de trabalho e infraestrutura urbana, levem em consideração o elevado e crescente contingente de trabalhadores pendulares no território nacional. 


\section{APÊNDICE}

Tabela A1. Estatísticas Descritivas

\begin{tabular}{|c|c|c|c|c|c|}
\hline Variáveis & Média & Desvio Padrão & Mínimo & Máximo & Observações \\
\hline $\begin{array}{l}\text { Taxa de entrada } \\
\text { pendular }\end{array}$ & 0.02 & 0.19 & 0.00 & 9.85 & 5564 \\
\hline $\begin{array}{l}\text { Distância até a } \\
\text { capital }\end{array}$ & 253.58 & 164.06 & 0.00 & 1476.28 & 5564 \\
\hline $\begin{array}{l}\text { Densidade } \\
\text { populacional }\end{array}$ & 108.21 & 572.47 & 0.13 & 13030.48 & 5564 \\
\hline IDHM - renda & 0.64 & 0.08 & 0.40 & 0.89 & 5564 \\
\hline IFDM - saúde & 0.67 & 0.16 & 0.08 & 1.00 & 5564 \\
\hline IFDM - educação & 0.68 & 0.13 & 0.25 & 1.00 & 5564 \\
\hline $\begin{array}{l}\text { Theil-L dos } \\
\text { rendimentos do } \\
\text { trabalho }\end{array}$ & 0.39 & 0.12 & 0.12 & 1.40 & 5564 \\
\hline
\end{tabular}

Fonte: elaboração própria.

Tabela A2. Determinantes dos fluxos pendulares no Brasil: Estimação dos modelos econométricos por MQO e pelo método generalizado dos momentos (GMM)

\begin{tabular}{|c|c|c|c|}
\hline \multirow[t]{2}{*}{$\begin{array}{l}\text { Variável dependente: } \\
\text { Taxa de entrada pendular }\end{array}$} & MQO & $\begin{array}{c}\text { Modelo com Lag } \\
\text { Espacial (SAR - } \\
\text { GMM) }\end{array}$ & $\begin{array}{c}\text { Modelo com Erro } \\
\text { Espacial (SEM - } \\
\text { GMM) }\end{array}$ \\
\hline & (1) & (2) & (3) \\
\hline \multirow[t]{2}{*}{ Constante } & 0.013 & -0.005 & 0.015 \\
\hline & $(0.029)$ & $(0.030)$ & $(0.029)$ \\
\hline \multirow[t]{2}{*}{ Distância até a capital $(\log )$} & $-0.055 * * *$ & $-0.053 * * *$ & $-0.055^{* * *}$ \\
\hline & $(0.003)$ & $(0.003)$ & $(0.003)$ \\
\hline
\end{tabular}


DETERMINANTES DOS MOVIMENTOS PENDULARES NO BRASIL: UMA ANÁLISE ESPACIAL

\begin{tabular}{|c|c|c|c|}
\hline Densidade populacional (log) & $0.012 * * *$ & $0.013 * * *$ & $0.012 * * *$ \\
\hline & $(0.002)$ & $(0.002)$ & $(0.002)$ \\
\hline \multirow[t]{2}{*}{ IDHM - renda } & $0.367 * * *$ & $0.382 * * *$ & $0.357 * * *$ \\
\hline & $(0.044)$ & $(0.044)$ & $(0.044)$ \\
\hline \multirow[t]{2}{*}{ IFDM - educação } & 0.008 & 0.018 & 0.013 \\
\hline & $(0.028)$ & $(0.029)$ & $(0.028)$ \\
\hline \multirow[t]{2}{*}{ IFDM - saúde } & $-0.069 * * *$ & $-0.066^{* * *}$ & $-0.072 * * *$ \\
\hline & $(0.020)$ & $(0.020)$ & $(0.020)$ \\
\hline \multirow[t]{2}{*}{$\begin{array}{l}\text { Theil-L dos rendimentos do } \\
\text { trabalho }\end{array}$} & $0.162 * * *$ & $0.151 * * *$ & $0.161 * * *$ \\
\hline & $(0.021)$ & $(0.021)$ & $(0.021)$ \\
\hline \multirow[t]{2}{*}{ W_TMIGPEND $(\rho)$} & & $-0.608 * * *$ & \\
\hline & & $(0.211)$ & \\
\hline \multirow[t]{2}{*}{$\lambda$} & & & 0.043 \\
\hline & & & $(0.053)$ \\
\hline $\mathrm{R}^{2}$ & 0.116 & & \\
\hline $\mathrm{R}^{2}$ Ajustado & 0.116 & 0.111 & 0.116 \\
\hline Observações & 5564 & 5564 & 5564 \\
\hline
\end{tabular}

Fonte: elaboração própria. Obs.: matriz de inversa da distância (raio de 60 km).

Notas: desvios-padrão entre parênteses. ${ }^{* * *}$ Estatisticamente significante a $1 \%$. ${ }^{* *}$ Estatisticamente significante a $5 \%$. *Estatisticamente significante a $10 \%$. 


\section{REFERÊNCIAS}

Almeida, E. S. (2012). Econometria Espacial Aplicada. Campinas: Alínea Editora. Ântico, C. (2005). Deslocamentos pendulares na região metropolitana de São Paulo. São Paulo em Perspectiva, 19(4), 110-120.

Anselin, L. (2013). Spatial econometrics: methods and models. Dallas: Springer Science \& Business Media.

Axisa, J. J., Scott, D. M., \& Newbold, K. B. (2012). Factors influencing commute distance: a case study of Toronto's commuter shed. Journal of Transport Geography, 24(C), 123-129.

Baller, R. D., Anselin, L., Messner, S. F., Deane, G., \& Hawkins, D. F. (2001). Structural covariates of U.S. county homicide rates: incorporating spatial effects. Criminology, 39(3), 561-588.

Brueckner, J. K. (1987). The structure of urban equilibria: A unified treatment of the Muth-Mills model. In E. S. Mills (Ed.), Handbook of regional and urban economics (Vol. 2, pp. 821-845). North Holland: Elsevier.

Cunha, J. M. P., Stoco, S., Dota, E. M., Negreiros, R. M. C., \& Miranda, Z. A. I. (2013). A mobilidade pendular na macro metrópole paulista: diferenciação e complementariedade sócio espacial. Cadernos Metrópole, 15(30), 433-459.

Dewey, J., \& Montes-Rojas, G. (2009). Inter-city wage differentials and intra-city workplace centralization. Regional Science and Urban Economics, 39(5), 602-609.

Florax, R. J., Folmer, H., \& Rey, S. J. (2003). Specification searches in spatial econometrics: the relevance of Hendry's methodology. Regional Science and Urban Economics, 33(5), 557-579.

IBGE. Instituto Brasileiro de Geografia e Estatística. Disponível em http://www. ibge.gov.br

IPEA. Instituto de Pesquisa Econômica Aplicada. Disponível em http://www.ipeadata.gov.br/

Jardim, A. P. (2011). Reflexões sobre a mobilidade pendular. In L. A. P. Oliveira, \& A. T. R. Oliveira (Eds.). Reflexões sobre os deslocamentos populacionais no Brasil (pp. 58-70). Rio de Janeiro: IBGE.

Jardim, A. P., \& Ervatti, L. (2009). Movimentos pendulares e circulares da população na região petrolífera do Estado do Rio de Janeiro: reflexões analíticas. Rio de Janeiro: IBGE.

Justo, W. R., Ferreira, R. A., Lima, C. F., \& Martins, G. N. (2010). Os Determinantes da Migração e da Migração de Retorno Intermunicipal no Brasil. In Anais do XXXVIII Encontro Nacional de Economia. Associação Nacional dos Centros de Pós-Graduação em Economia, Salvador, Brasil. Disponível 
em http://www.anpec.org.br/encontro2010/inscricao/arquivos/000-2e5e49a$5 \mathrm{fb} 7 \mathrm{~d} 7 \mathrm{~b} 1369 \mathrm{~d} 25 \mathrm{a} 6273 \mathrm{da} 1 \mathrm{a} 76 . \mathrm{pdf}$

Justo, W. R., \& Silveira Neto, R. D. M. (2008). O que determina a migração interestadual no Brasil?: um modelo espacial para o período 1980-2000. Revista Econômica do Nordeste, 39(4), 428-447.

Kelejian, H. H., \& Prucha, I. R. (1998). A Generalized Spatial Two-Stage Least Squares Procedure for Estimating a Spatial Autoregressive Model with Autoregressive Disturbances. The Journal of Real Estate Finance and Economics, 17(1), 99-121. Disponível em https://doi. org/10.1023/A:1007707430416

Kelejian, H. H., \& Prucha, I. R. (1999). A generalized moments estimator for the autoregressive parameter in a spatial model. International economic review, 40(2), 509-533.

Krugman, P. (1998). What's new about the new economic geography? Oxford review of economic policy, 14(2), 7-17.

Lloyd, C., \& Shuttleworth, I. (2005). Analysing commuting using local regression techniques: scale, sensitivity, and geographical patterning. Environment and Planning, 37(1), 81-103.

Lobo, C. (2016). Mobilidade pendular e a dispersão espacial da população: evidências com base nos fluxos com destino às principais metrópoles brasileiras. Caderno de Geografia, 26(45), 285-298.

Lösch, A. (1954). The economics of location. (2a ed.) New Haven: Yale University Press.

Mata, D., Oliveira, C. W., Pin, C., \& Resende, G. M. (2007). Quais Características das Cidades Determinam a Atração de Migrantes Qualificados? (IPEA, Texto para Discussão, No. 1305). Brasília: Instituto de Pesquisa Econômica Aplicada. Disponível em http://repositorio.ipea.gov.br/bitstream/11058/1411/1/TD_1305.pdf

McLafferty, S. (1997). Gender, race, and the determinants of commuting: New York in 1990. Urban geography, 18(3), 192-212.

Mills, E. S. (1972). Studies in the Structure of the Urban Economy. Baltimore: Johns Hopkins Press.

Mingoti, S. A. (2007). Análise de Dados Através de Métodos de Estatística Multivariada - Uma Abordagem Aplicada. Belo Horizonte: Editora UFMG.

Miranda, R. A., \& Domingues, E. P (2008). Nova Economia Urbana e Movimento Pendular na Região Metropolitana de Belo Horizonte. In Anais do XIII Seminário sobre a Economia Mineira. Centro de Desenvolvimento e Planejamento Regional. Diamantina, Mina Gerais. 
Moura, R., Castello Branco, M. L. G., \& Firkowski, O. L. C. (2005). Movimento pendular e perspectivas de pesquisas em aglomerados urbanos. São Paulo em perspectiva, 19(4), 121-133.

Moura, R., Delgado, P., \& Costa, M. A. (2013). Movimento pendular e políticas públicas: algumas possibilidades inspiradas numa tipologia dos municípios brasileiros. In R. Boueri, \& M. A. Costa (Eds.). Brasil em desenvolvimento 2013: estado, planejamento e políticas públicas (pp. 665-696). Brasília: IPEA.

Muth, R. F. (1969). Cities and housing : the spatial pattern of urban residential land use. Chicago: University of Chicago Press.

Nowotny, K. (2011). Commuting, residence and workplace location attractiveness and local public goods. Scienze Regionali, 10(1), 109-136.

Papanikolaou, G. (2006). Spatial and Individual Influence on Commuting Behaviour in Germany. In Anais do 46th Congress of the European Regional Science Association. Volos, Greece, European Regional Science Association. Disponível em https://www.econstor.eu/bitstream/10419/118378/1/ ERSA2006_468.pdf

Pereira, R. H. M., \& Herrero, V. (2009). Mobilidade Pendular: uma proposta teórico-metodológica. (IPEA, Texto para Discussão No.1395). Rio de Janeiro: Instituto de Pesquisa Econômica Aplicada. Disponível em http://repositorio. ipea.gov.br/handle/11058/1656

Programa das Nações Unidas para o Desenvolvimento. (2013). Atlas do Desenvolvimento Humano no Brasil. ( $3^{\mathrm{a}}$ ed.). Brasília: PNUD, Ipea, FJP.

Sandow, E., \& Westin, K. (2010). The persevering commuter-Duration of long-distance commuting. Transportation Research Part A: Policy and Practice, 44(6), 433-445.

So, K. S., Orazem, P. F., \& Otto, D. M. (2001). The effects of housing prices, wages, and commuting time on joint residential and job location choices. American Journal of Agricultural Economics, 83(4), 1036-1048.

Van Ommeren, J., Rietveld, P., \& Nijkamp, P. (1997). Commuting: In Search of Jobs and Residences. Journal of Urban Economics, 42(3), 402-421.

Von Thünen, J. H. (1966). The isolated state. New York: Pergamon Press.

(C) 2021 por los autores; licencia no exclusiva otorgada a la revista Estudios económicos. Este artículo es de acceso abierto y distribuido bajo los términos y condiciones de una licencia Atribución-No Comercial 4.0 Internacional (CC BY-NC 4.0) de Creative Commons. Para ver una copia de esta licencia, visite http://creativecommons.org/licenses/by-nc/4.0 